ORIGINAL RESEARCH ARTICLE

\title{
Making Abortion Safer in Rwanda: Operationalization of the Penal Code of 2012 to Expand Legal Exemptions and Challenges
}

\author{
Nuriye Nalan Sahin Hodoglugil ${ }^{1}$, Fidele Ngabo ${ }^{2}$, Joanna Ortega ${ }^{1}$, Laetitia Nyirazinyoye ${ }^{3}$, \\ Eugene Ngoga ${ }^{4}$,Evangeline Dushimeyezu ${ }^{1}$, Eugene Kanyamanza ${ }^{2}$ and Ndola Prata ${ }^{4}$
}

University of California at Berkeley, School of Public Health, Bixby Center for Population, Health and Sustainability, Berkeley, CA, USA ${ }^{1}$; Rwanda Ministry of Health, Kigali, Rwanda ${ }^{2}$; University of Rwanda College of Medicine and Health Sciences, School of Public Health Kigali, Rwanda ${ }^{3}$; Rwanda Society of Obstetricians and Gynecologists ${ }^{4}$

*For Correspondence: E-mail: Nuriye.hodoglugil@gmail.com; Phone: +1 5102203060

\begin{abstract}
Penal code was revised in Rwanda in 2012 allowing legal termination of pregnancy resulting from rape, incest, forced marriage, or on medical grounds. An evaluation was conducted to assess women's access to abortion services as part of an ongoing program to operationalize the new exemptions for legal abortion. Data was collected from eight district hospitals; seven genderbased violence (GBV) centers and six intermediate courts. Three focus group discussions and 22 in-depth interviews were conducted with key informants. At hospitals, of the 2,644 uterine evacuation records (July 2012-June 2014), and 312 monitoring cases (August-December 2014), majority of all uterine evacuations (97\% and 85\% respectively, for the two periods) were for obstetric conditions, and induced abortion on medical grounds accounted for $2 \%$ vs. $15 \%$ respectively. Medical abortion was the prominent method of uterine evacuation. At the GBV centers, 3,763 records were identified retrospectively; 273 women were pregnant. Since the legal reform there was only one abortion for a pregnancy resulting from rape. Abortion stigma and court order requirement are major barriers to access services. The operationalization program has made significant contributions to make abortion safer in Rwanda but this evaluation demonstrates that further work is required to reach the goal of providing safe abortion services to all eligible women. Addressing abortion stigma at the community, organizational and structural levels; further strengthening of service provision; and streamlining legal requirements to protect particularly young women from sexual violence and making abortion a realistic option for GBV victims are some of the important next steps. (Afr J Reprod Health 2017; 21[1]: 82-92).
\end{abstract}

Keywords: Rwanda, abortion, law, rape, gender-based violence, stigma

\section{Résumé}

Le code pénal a été révisé au Rwanda en 2012, ce qui autorise l'interruption légale de la grossesse occasionnée par le viol, l'inceste, le mariage forcé ou pour des raisons médicales. Une évaluation a été menée pour évaluer l'accès des femmes aux services d'avortement dans le cadre d'un programme en cours visant à rendre opérationnelles les nouvelles exemptions pour l'avortement légal. Les données ont été recueillies auprès de huit hôpitaux de district; sept centres de violence sexiste (CVS) et six cours intermédiaires. Trois discussions à groupe témoin et 22 entretiens en profondeur ont été menés avec des informateurs clés. Dans les hôpitaux, sur les 2644 registres d'évacuation utérine (juillet 2012-juin 2014) et 312 cas de surveillance (aoûtdécembre 2014), la majorité des évacuations utérines (respectivement $97 \%$ et $85 \%$ pour les deux périodes) étaient pour des problèmes obstétricaux, et l'avortement provoqué pour raisons médicales représentaient $2 \%$ contre $15 \%$ respectivement. L'avortement médical était la méthode primordiale d'évacuation de l'utérine. Aux centres CVS, 3763 enregistrements ont été identifiés rétrospectivement; 273 femmes étaient enceintes. Depuis la réforme légale, il n'y a eu qu'un avortement pour une grossesse en raison d'un viol. La stigmatisation de l'avortement et l'exigence de la décision du tribunal sont des obstacles majeurs aux services d'accès. Le programme d'opérationnalisation a contribué de manière significative à rendre l'avortement plus sûr au Rwanda, mais cette évaluation démontre qu'un travail supplémentaire est nécessaire pour atteindre l'objectif de fournir des services d'avortement sécurisé à toutes les femmes admissibles. Aborder la stigmatisation de l'avortement au niveau communautaire, organisationnel et structurel; le renforcement de la prestation de services; la rationalisation des exigences légales pour protéger particulièrement les jeunes femmes contre les violences sexuelles et faire de l'avortement une option réaliste pour les victimes de la CVS sont parmi les prochaines démarches importantes à suivre. (Afr J Reprod Health 2017; 21 [1] : 82-92).

Mots-clés: Rwanda, avortement, droit, viol, violence sexiste, stigmatisation 


\section{Hodoglugil et al.}

\section{Introduction}

Rwanda, "land of a thousand hills" with a population of about 11.5 million $^{1}$ is one of the few countries in the African continent to reach the fifth millennium development goal $^{2}$, where the maternal mortality rate decreased substantially from 1,071 in 100,000 live births in 2000 to 210 in $2015^{3}$. However, unwanted pregnancies and unsafe abortions continue to threaten women's health. Based on a recent report, nearly half of all pregnancies were unintended; $22 \%$ of those ended in induced abortion; and half of all abortions were performed by untrained individuals, making them very high risk ${ }^{4}$. Furthermore, treating complications of unsafe abortions is quite costly for the health system, adding up to a significant share of total expenditure in reproductive health ${ }^{5}$.

In Africa overall, at least $9 \%$ of maternal deaths are due to unsafe abortion; about 1.6 million women are treated annually for complications from unsafe abortion; and $90 \%$ of women of childbearing age live in countries with restrictive abortion laws ${ }^{6,7}$. Where abortion is restricted, more women resort to clandestine procedures, which are often unsafe ${ }^{7}$, leading to abortion related complications and mortality ${ }^{8}$. Revising abortion laws is one important step to allow women gain access to safe and legal services. While evidence is limited, it is increasingly being shown that liberalized laws are followed by improved health outcomes for women. ${ }^{9}$ In South Africa, for instance, a dramatic reduction in abortion-related maternal deaths in facilities was associated with the revised law; and in Ethiopia, complications from unsafe abortion had declined based on a subnational study?

While Rwanda does not have an "abortion law", during the revisions of the Penal Code in 2012, articles related to abortion were also revised, supported by advocacy groups in the country ${ }^{10}$. The Penal Code of 2012 (PC-2012) included four exemptions from criminal liability for abortion ${ }^{11}$ and lifted the reservations to Article 14(2)(c) of the African Charter on Human and People's Rights of Women in Africa ${ }^{12}$. According to the PC-2012 a pregnancy can be terminated on medical grounds

\section{Legal Abortion in Rwanda}

(i.e. when the pregnancy "severely jeopardizes the health of the unborn baby or that of the pregnant woman"); or if the pregnancy is a result of rape, incest or forced marriage (Table 1$)^{11}$. With the exception of pregnancy termination on medical grounds, women are required to obtain a court order before abortion can be performed at a health facility.

Accelerating the reduction of maternal morbidity and mortality has been a major goal of the Rwandan Ministry of Health $(\mathrm{MOH})^{13}$, and unsafe abortion has been addressed through policy and programmatic activities since 2010. A National Postabortion Care (PAC) program was successful in expanding PAC services to primary level facilities through the use of misoprostol and manual vacuum aspiration, with the involvement of mid-level providers ${ }^{14,15}$. The PAC program was then followed by the Operationalization of the Penal Code of 2012 Program (hereafter referred to as the PC-2012 Program) launched in January 2013, aiming to increase accessibility of safe abortion services within the legal framework.

A five-year strategic plan was developed by the MOH and partners to operationalize the PC2012, which set two major goals: provision of safe abortion services to $100 \%$ of women in need and eligible according to the legal framework; and reduction of morbidity and mortality associated with unsafe abortion by $75 \%$ by $2018^{16}$. A National PC-2012 Protocol was developed with the involvement of stakeholders in the country through a re-iterative and consultative process, which further detailed the procedures for service provision, taking into account the medical, legal and psycho-social aspects of abortion services ${ }^{17}$. Because three exemptions (rape, incest and forced marriage) are classified as gender based violence (GBV), the PC-2012 Protocol linked GBV management with legal abortion services. Medicines for uterine evacuation (misoprostol and mifepristone) were registered in Rwanda and made available at facilities as part of the program.

Following development of the Protocol and training materials for the PC-2012 Program, 24 health care providers (obstetrician/gynecologists, physicians, nurses, 
Table 1:Legal Framework for Abortion in Rwanda Based on the Penal Code of $2012^{11}$

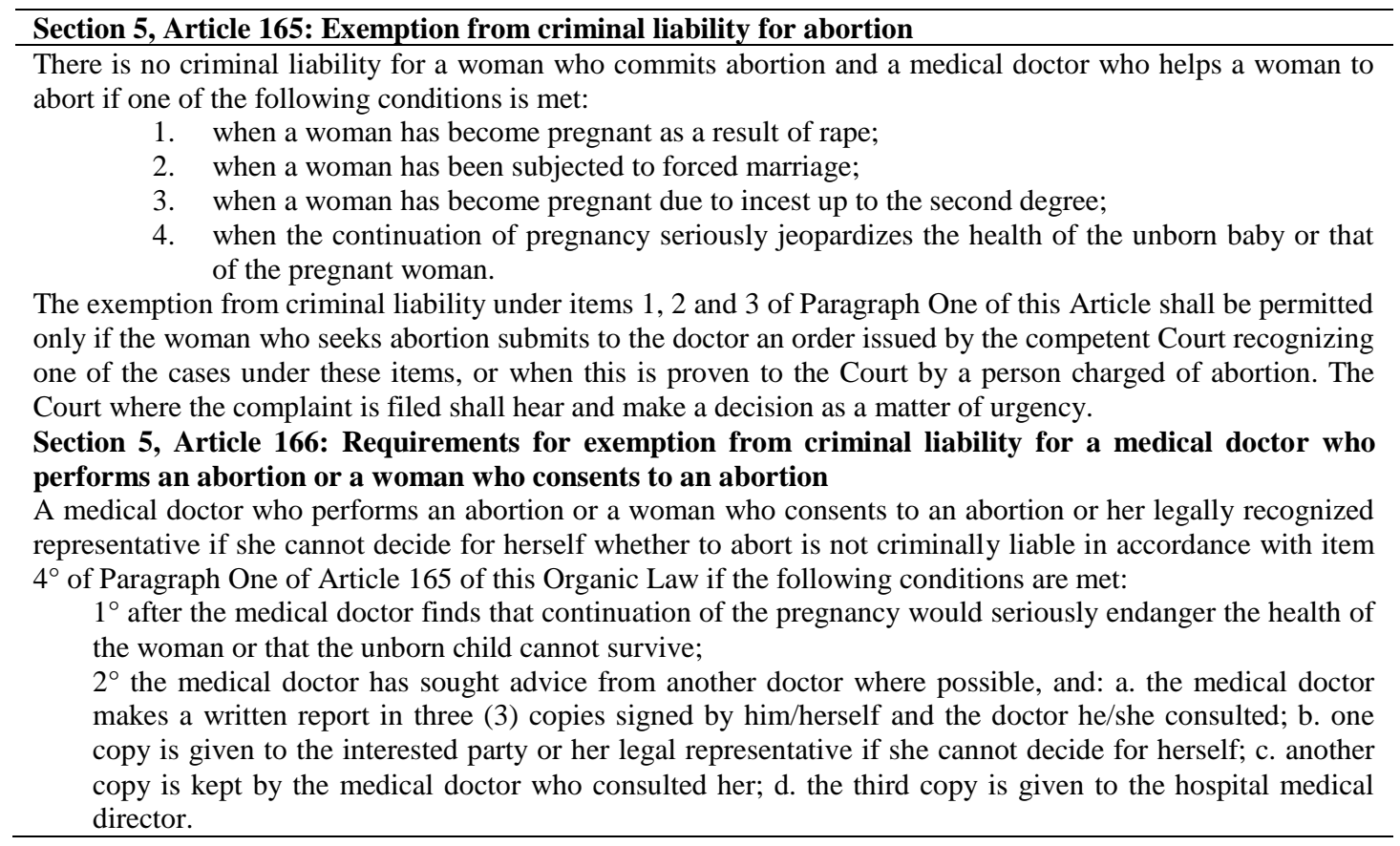

midwives and counselors) were trained in mid2014 by the $\mathrm{MOH}$ in collaboration with Rwanda Society of Obstetricians and Gynecologists and other partners. The training included values clarification and attitude transformation activities to address abortion stigma; postabortion family planning; medical and surgical methods of uterine evacuation; management of complications; forensic examination for rape; psycho-social counseling for abortion clients; and monitoring and evaluation of abortion-related services. Orientation meetings were also conducted in each one of the eight initial sites (for a total of 138 participants) to inform service providers, administrators, other facility staff, as well as district level representatives of courts and Rwanda National Police (RNP) on the legal framework and the PC-2012 Program. Community meetings were held to inform community leaders, reaching a total of 440 participants. Educational brochures and key questions were used to cover information on the PC-2012, GBV and pregnancy termination services. Participants also shared cultural perspectives, discussed concerns and ways to inform their larger communities.

Within this background, an evaluation was conducted between June-December 2014 to identify the status of abortion services in the country since the publication of the Penal Code in 2012. We present here the results from this evaluation, and to our knowledge, this is the first published research on provision of abortion services through public facilities in Rwanda.

\section{Methods}

The evaluation included data from eight district hospitals (Central Hospital of University of Kigali [CHUK], Gihundwe Hospital, Kabutare Hospital, Kacyiru Police Hospital, Muhima Hospital, Nyagatare Hospital, Rwanda Military Hospital, and Ruhengeri Hospital); and seven GBV centers affiliated with these facilities (one of the facilities did not have a GBV center). GBV centers were included because they are designed as One-StopCenters to serve as point of entry for the survivors, and provide care and support such as counseling, assistance with legal procedures and safe houses. GBV centers also provided referrals to the district hospital for further medical care as needed ${ }^{18}$. Six intermediate courts in the program areas were also included in the evaluation to inquire about issuing of court orders for abortion. 


\section{Hodoglugil et al.}

Because the PC-2012 was published in June 2012, and the evaluation was conducted in July 2014 as part of an ongoing program, quantitative data on service provision at the facilities was collected separately to capture the situation before the program started (July 2012-June 2014; i.e. "retrospective period"), and during monitoring of the program (August-December 2014; i.e. "prospective period"). For the July 2012-June 2014 period, facility records for abortion services were reviewed retrospectively, and data was extracted on tools specifically developed for the evaluation. For the August-December 2014 period, data was collected prospectively with routine monitoring tools developed for the PC-2012 Program. All data was collected by a trained data manager in each facility (chief obstetric nurse in hospitals, and GBV focal person at GBV centers). Data collection tools from facilities were sent to Kigali program office, and entered electronically into a database after data cleaning and clarification of irregularities.

At each one of the intermediate courts serving program districts, paper and electronic records between July 2012 and June 2014 were reviewed thoroughly by trained data collectors (law students) to identify any court order to terminate pregnancy as a result of rape, incest or forced marriage. A review of records at the prosecution offices was planned initially, but was not possible due to lack of time to get additional approvals.

For the qualitative inquiry, three focus group discussions (FGD) were conducted with 33 women (aged 18-45) recruited from Female Students Association and Women's Association in Kigali City, and Women's Association in Musanze between July-August 2014. FGD were conducted in Kinyarwanda, tape recorded, and key points were summarized in English. In-depth interviews were conducted with key informants including health care providers from hospitals and GBV Centers (12); representatives of courts (6); Ministry of Justice (MOJ) (1); MOH (1) and civil society organizations (2) in English, French and/or Kinyarwanda depending on the preference of the respondent. Interviews were tape recorded if the respondent consented. Otherwise, extensive notes

\section{Legal Abortion in Rwanda}

were taken which were transcribed and translated into English. Qualitative data was analyzed by the inter-disciplinary qualitative research team based in Rwanda and the US. The team held discussions to clarify and agree on the key themes that emerged from the data in relation to perspectives on abortion and implementation of the PC-2012. Research protocol for the evaluation was approved by the Rwanda National Ethics Committee.

\section{Results}

\section{Quantitative findings from hospitals}

A total of 2,644 records were identified for pregnancy termination in the retrospective record review (July 2012-June 2014), and prospective data was collected from 312 women during the six months of monitoring (August-December 2014) at the eight hospitals (Figure 1). It was found that "abortion" did not exclusively mean "termination of an intra-uterine pregnancy with a live fetus" in the minds of practitioners and in the records. Most records of "abortion" were actually procedures to treat other obstetric conditions including intrauterine fetal death (IUFD), missed abortion, molar pregnancy, and sometimes ectopic pregnancy. (Incomplete abortion or miscarriage cases were recorded separately in PAC registers, and not included in abortion records). Based on this categorization, "other obstetric reasons" accounted for the majority of "abortion" cases, both based on retrospective record review (97\%) and monitoring (85\%) data (Figure 1).

In terms of legal termination of a pregnancy with a live fetus, all but one case was pregnancy termination on medical grounds. For the retrospective period, $1 \%$ of all 2,644 records indicated saving mother's health as the reason for induced abortion and another $1 \%$ was due to fetal impairment. During the monitoring period, on the other hand, $14 \%$ of 311 procedures were induced abortions to save the health of mother, and another $1 \%$ was for fetal impairment (data was missing for one women on the reason for termination). There was only one case of induced abortion where pregnancy was as a result of rape. This was the only record identified in hospitals- and triangulated with GBV and court data- between 


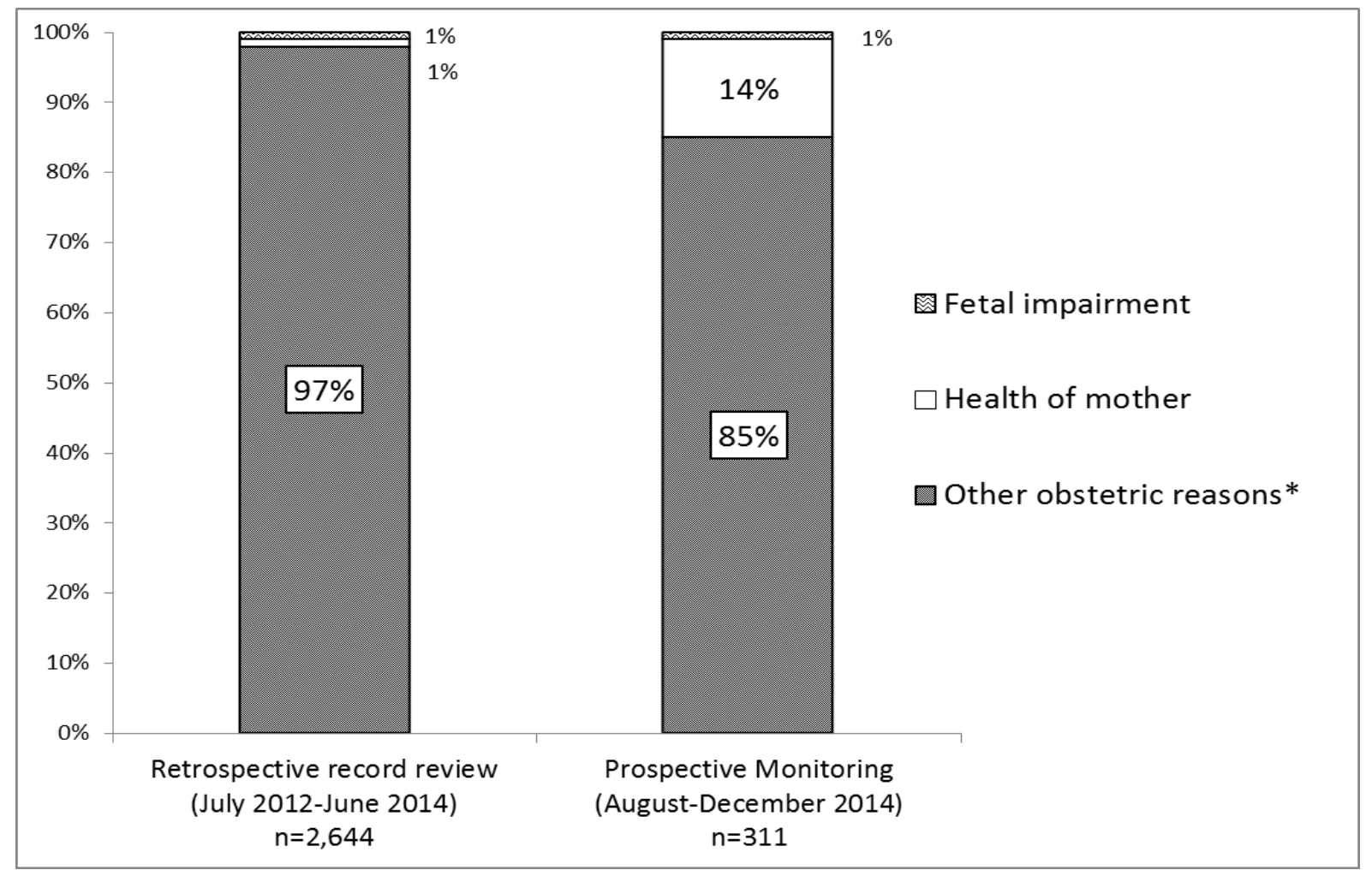

Figure 1: Reasons for Pregnancy Termination at District Hospitals Based on Data from Retrospective Record Review (July 2012-June 2014) and Monitoring (August-December 2014) Periods

*Other obstetric reasons include: intrauterine fetal death, missed abortion, molar pregnancy, trophoblastic disease, ectopic pregnancy and unspecified cases.

July 2012 and December 2014. It was a case of a minor, who had a court order proving rape and received medical abortion in April 2014.

Overall, medication methods were used for the majority of the uterine evacuation procedures, with $43 \%$ of all cases in the retrospective period and $64 \%$ in the monitoring period (Figure 2). The use of dilatation and curettage (D\&C) declined from almost $40 \%$ of all procedures in the retrospective records to $1 \%$ in the monitoring period.

Data on post abortion contraception, complications and side effects was available only for monitoring period. Of 312 women, $82 \%$ received post abortion contraceptive counseling and 26\% received a method before discharge (Table 2). Majority (87\%) of women did not experience any side effects or complications before discharge, and approximately $11 \%$ of women were reported to experience a side effect that was managed at the facility and $3 \%$ were referred for additional treatment (Table 2). One maternal death was reported in a patient who was admitted for IUFD and the postmortem examination concluded the reason of death as hemorrhagic shock secondary to uterine rupture.

\section{Findings from GBV centers}

Retrospective record review identified 3,763 survivors who sought GBV services after sexual violence. While 273 and 181 of these women were recorded as pregnant at the first and second visit respectively, GBV registries did not allow for a distinction of what proportion of these pregnancies were as a result of the sexual assault, and what proportion was assault on pregnant women. Therefore, it was not possible to identify how many women became pregnant as a result of rape. Among pregnant women who sought GBV services, 11 records showed a request to terminate 


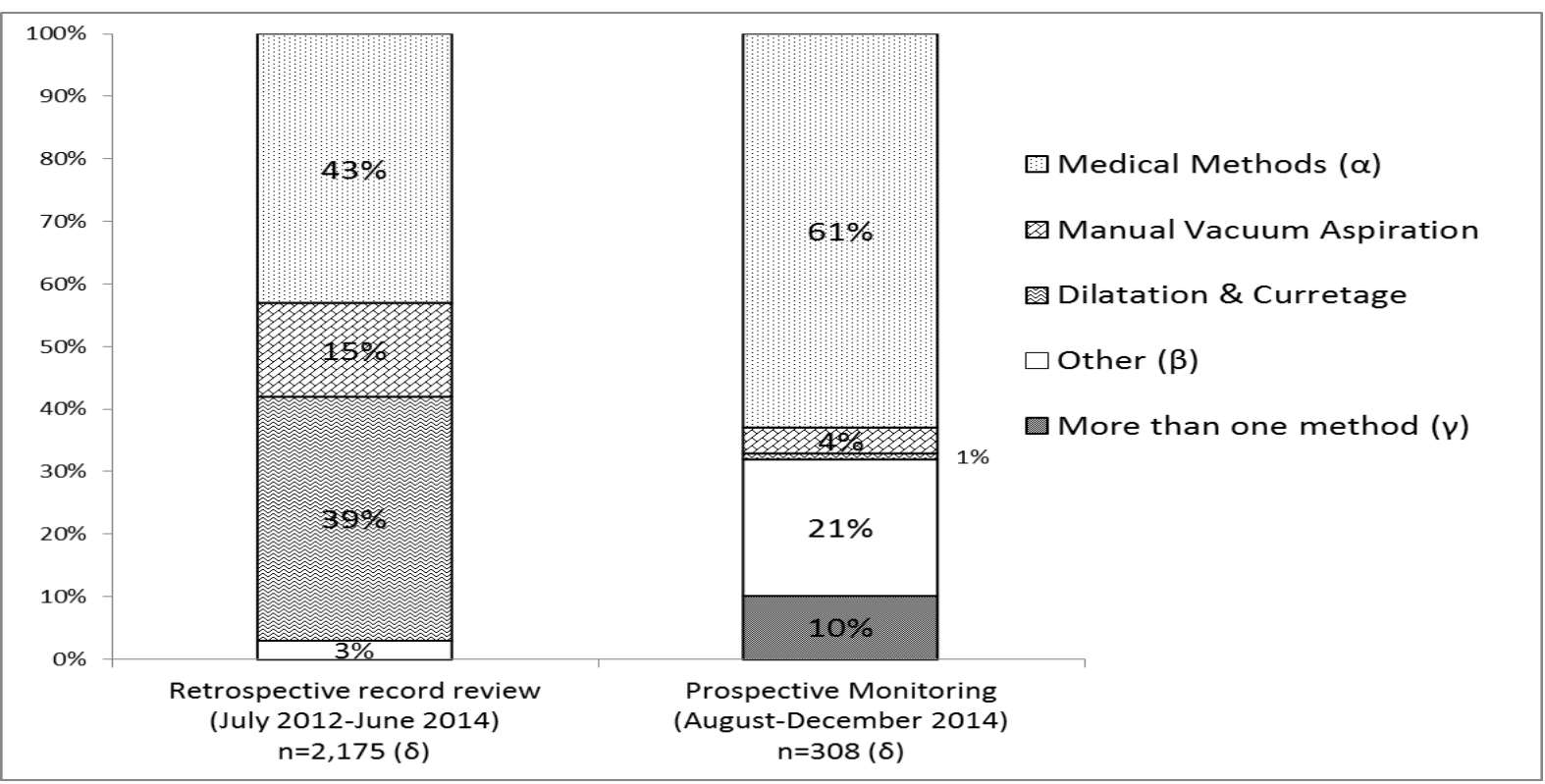

Figure 2: Distribution of Methods Used for Termination of Pregnancy Based on Data from Retrospective Record Review (July 2012-June 2014) and Monitoring (August-December 2014) Periods.

( $\alpha$ ) In the retrospective period, only misoprostol was available. During monitoring, of the 197 cases treated medically, 4 were with misoprostol-mifepristone combination and 193 were misoprostol alone.

( $\beta$ ) Other methods include: oxytocin, electric aspiration, dilatation and evacuation, cesarean section, and vaginal delivery.

$(\gamma)$ Data on the use of more than one method was only collected during monitoring.

( $\delta$ ) Data on pregnancy termination was not available for 469 cases in the retrospective period and for 3 cases during monitoring period.

Table 2: Postabortion Contraceptive Services and Complications and Side Effects Experienced by Uterine Evacuation Clients During Monitoring Period (August-December 2014)

\begin{tabular}{lll}
\hline Postabortion contraceptive service uptake $(\mathbf{n = 3 1 2})$ & No & \% \\
\hline Received contraceptive counseling & 250 & 82 \\
Did not receive contraceptive counseling & 62 & 18 \\
Did not get a contraceptive & 203 & 65 \\
Left the facility with a contraceptive & 109 & 35 \\
Distribution of contraceptive method used $(\mathbf{n = 1 0 9 )}$ & $\mathbf{N o}$ & $\%$ \\
Injectables & 29 & 27 \\
Condoms & 24 & 22 \\
Pills & 23 & 21 \\
Implants & 16 & 15 \\
IUD & 13 & 12 \\
Tubal ligation & 1 & 1 \\
Cycle beads & 1 & 1 \\
Side effects and complications before discharge among women & $\mathbf{N o}$ & $\%$ \\
seeking pregnancy termination services $(\mathbf{n}=\mathbf{3 1 2})$ & & \\
No side effects or complications & 270 & 86.5 \\
Abdominal pain & 21 & 6.7 \\
Severe bleeding & 10 & 3.2 \\
Abdominal pain and severe bleeding & 1 & 0.3 \\
Infection & 1 & 0.3 \\
Referred to another facility for additional treatment & 8 & 2.6 \\
Maternal death & 1 & 0.3 \\
\hline
\end{tabular}


the pregnancy, and of these women, only one presented a court order for abortion and received an abortion (the same case reported in hospital data above). There was no further information in the records about other pregnant women.

Between August-December 2014, 550 survivors of sexual violence sought GBV services, and of those $527(96 \%)$ were female, and 84\% were minors who were 18 years old or younger. A total of 84 survivors $(16 \%)$ were recorded to be pregnant as a result of rape $(96 \%)$, incest $(1 \%)$, or forced marriage (2\%); and four women were reported to have asked to terminate the pregnancy. Women who were pregnant were lost to follow-up.

\section{Data from the courts}

A review of records at the six courts serving eight program districts revealed that since the Penal Code was published in June 2012, there had been only one identified case of an approved court order, the same one triangulated with hospital and GBV data, issued by the Nyarugenge Intermediate Court in April 2014.

\section{Findings from qualitative inquiry}

Qualitative inquiry revealed some important insights to understand some of the barriers for accessing safe abortion services and some exemplary quotes from in-depth interview respondents are provided in Table 3. One key theme was the pervasiveness of the culture of silence and stigma around rape and abortion. Respondents in all groups felt that stigma was so strong that many survivors of rape would prefer to remain silent rather than seeking immediate help (Table 3, quote 1), and sometimes those who are eligible for legal abortion may still resort to unsafe abortion to avoid going to court and becoming public (Table 3, quote 2). Furthermore, disclosure of rape would cause them to face severe consequences such as being marginalized, shamed, and losing prospects of marriage. While there seemed to be some acceptance of women terminating a pregnancy as a result of rape, opposing views were also held that getting an abortion after a rape just made it worse and more stigmatizing (Table 3 , quote 3 ).
Another major theme was about the challenges to follow the legal procedures to get a court order to prove that the pregnancy was as a result of rape, incest or forced marriage (Table 3 quotes 4-6). Many times victims and/or their families would come forward only when a sexual assault resulted in a pregnancy, which was too late with the current legal framework to get a court order (Table 3, quote 4). According to the current GBV procedures, a victim of sexual assault has to report to a GBV center within 72 hours of the incident to get on records, and initiate the legal process. Health care providers felt that their hands were tied without a court order, while legal officials emphasized that they had to follow the law to confirm the evidence and convict the rapist before a court order can be issued. Some legal officials did not see a problem with the requirement to admit to a GBV center within the first 72 hours of the sexual assault, and felt that women should be able to follow the routine GBV management procedures, and get a court order if needed. Women admitting to a GBV center later with a pregnancy claiming that it was a result of rape were mostly seen as trying to "abuse" the system, and hence their chances were very slim -if not impossible- to get a court order. The opinion that the victims should be able to seek justice immediately was partially grounded in the perception that rape was an acute, one time condition. However, FGD data did not support these assumptions, where women shared stories of young women who were repeatedly assaulted over many years, and anecdotes of incest cases, where victims continued to live with their perpetrators.

It was also mentioned that even if a woman might have initiated the legal process of getting a court order, the time it takes to complete the procedures could still make it too late to get an abortion, and a few anecdotes were cited during the FGD of this happening, including a minor giving birth to a child before her family was able to get a court order (Table 3, quote 6).

While some GBV counselors were not trained on the PC-2012 and did not offer abortion as an option, some others, who were informed of the exemptions still counseled the survivors to accept the pregnancy as a way to cope with the 
Table 3: Major Themes and Exemplary Quotes from the In-depth Interview Respondents*

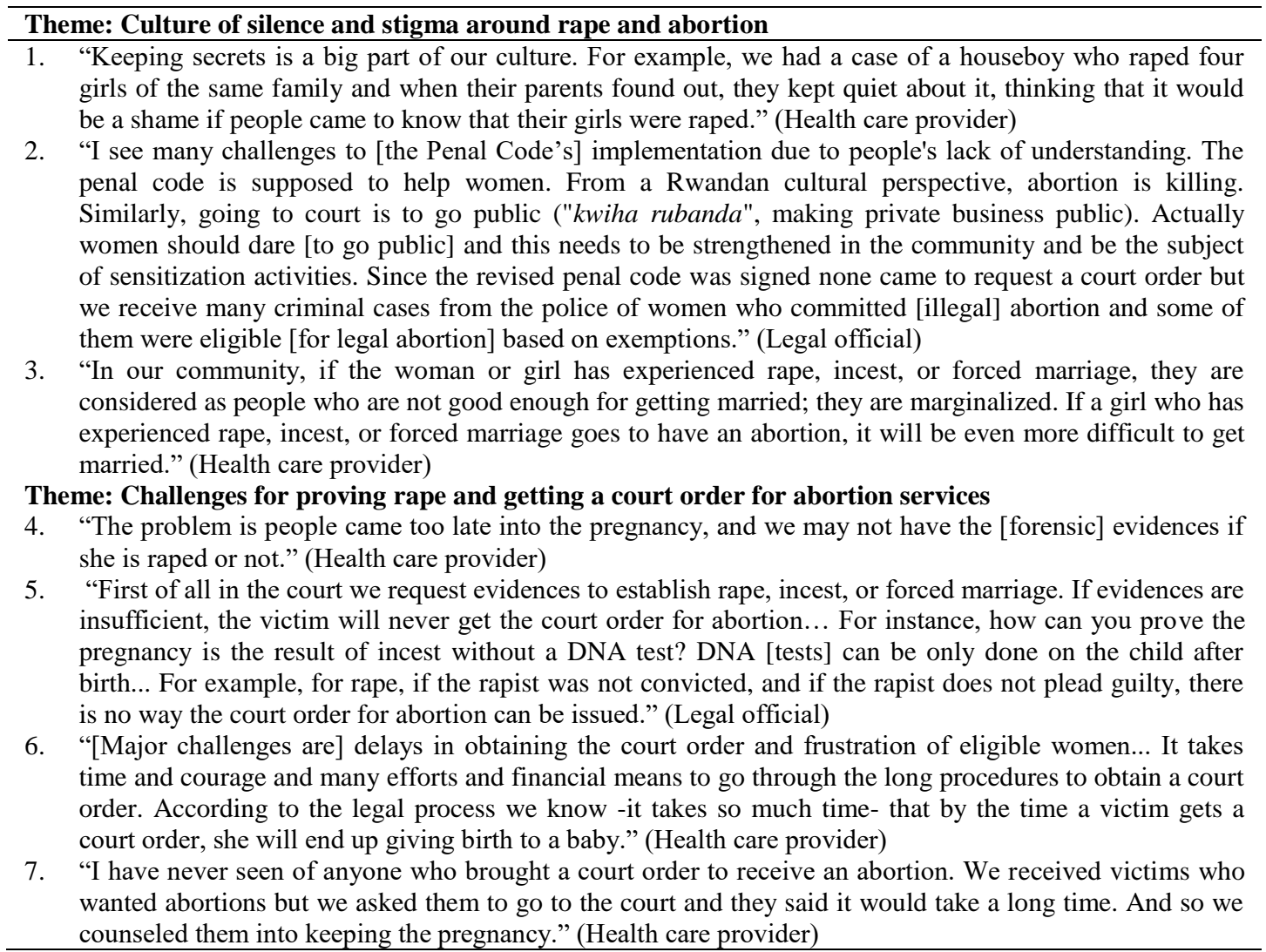

*Each quote belongs to a different respondent, and further identifiers such as district or facility was removed to keep confidentiality.

trauma, acknowledging the difficulty of getting a court order (Table 3, quote 7). Some health care providers and FGD respondents were concerned that women who are eligible for exemptions would still resort to unsafe abortion rather than seeking a legal termination due to the culture of silence and stigma, coupled with the difficulty of getting a court order.

Severe consequences were also listed for women who delivered a child conceived from a rape including rejection by their families and society, being expelled from school, and financial hardship. Children born to survivors were stigmatized (ikinyendaro; "bastard"), with increased risk of child neglect and abuse.

\section{Discussion}

Rwanda is exceptional in that, even though the
PC-2012 is still quite restrictive from a global perspective, decision makers were willing to take the step to operationalize and implement the new exemptions. Only a handful of countries have liberal abortion laws in Africa ${ }^{7}$, and it has been reported previously that easing the legal restrictions on abortion does not automatically translate into access to safe abortion services ${ }^{9}$. The process requires further actions over several years, including: informing the public and the providers about the reform; promoting low-cost and safe abortion techniques; involving midlevel providers in service provision; and, anticipating and planning for a backlash against abortion law reforms 9 . The priority Rwandan $\mathrm{MOH}$ placed on further reduction in maternal mortality through addressing unsafe abortions is somewhat rare in the region and needs to be recognized for the 
several successful steps of its implementation. However, one of the main findings of this evaluation -that there was only one termination of a pregnancy as a result of rape in the two and a half year period- attests to the significant barriers women are facing to access the services.

Qualitative inquiry showed the pervasiveness of abortion stigma and how difficult it was for a victim of rape to speak up and seek care. Abortion stigma has been conceptualized to manifest at different levels, including individual, community, organizational/institutional, governmental/structural, and framing discourses and mass culture levels; and negatively impact women's health ${ }^{19}$. While it is already stigmatizing for a woman to be raped, even though she is the victim, she gets further stigmatized if she seeks abortion. This compound stigma is a powerful inhibiting force leading to severe consequences for women, including death, further abuse of victims, and poor quality of care ${ }^{19}$. Similarly, a study from Kenya also highlighted abortion related stigma as a key driver in silencing women's ability to seek help, and suggested that in communities where abortion stigma is higher, women are more likely to resort to unsafe abortion, even when legal options are available ${ }^{20}$.

While this evaluation was not designed as a before/after study, and monitoring data was collected in the midst of ongoing initiatives to address unsafe abortions, it is important to highlight some of the differences observed between the two data collection periods to demonstrate the trajectory in the two and a half years. As for the differences in the reasons for pregnancy termination (abortion on medical grounds accounting for $2 \%$ of all procedures in retrospective record review vs. $15 \%$ during monitoring), some of the differences can be attributed to the different recording systems. Monitoring period followed training of providers in the eight program sites, who were familiarized with the PC-2012 Protocol, and were trained on data collection tools, so monitoring data is expected to be more accurate than retrospective record data. On the other hand, it is also plausible that fewer abortion procedures were done on medical grounds in the retrospective period and/or more acceptable categories (e.g. obstetric reasons) could have been used in the records as many providers were not fully informed about the Penal Code of 2012 until training and orientation activities in 2014.

The substantial decline in the use of $\mathrm{D} \& \mathrm{C}$ (39\% retrospectively vs. $1 \%$ prospectively) can be attributed to the training on the use of misoprostol and MVA throughout the preceding PAC program. Misoprostol tablets and MVA kits were distributed throughout the country, and the initial phase of the national PAC program showed $83 \%$ use of misoprostol for the treatment of incomplete abortion ${ }^{14}$. This difference can be interpreted as a sign of successful transition to safer methods of uterine evacuation in the facilities included.

It was not possible to draw conclusions about women's choices to keep or terminate a pregnancy from the retrospective data at the GBV centers, as the information was not captured in the registers. Tools used during prospective monitoring, on the other hand, included information on pregnancies as a result of GBV, where a handful of women were recorded as having made a request to terminate their pregnancies. However, the forms were completed at the end of each session with a client, and there is no way of knowing how the counseling session might have influenced women's final choices on abortion, especially if keeping the pregnancy was provided as the key message. Because many providers counseled on keeping the pregnancy, it is likely that the number of women who might have initially preferred to terminate their pregnancies is underestimated.

The majority of pregnant GBV survivors were minors (age <18), for whom specialized youth-friendly services were not available. Since child defilement (defined as "any sexual act with a child" in article 190 of the PC-2012) was not included specifically as an exemption for abortion, this issue remains to be resolved in the future to support further advancement of the rights of young people in the country. One comparable example is from Ethiopia, where penal code was revised in $2005^{21}$. Ethiopia's legal framework for abortion is very similar to that of Rwanda's, and the main differences are that: abortion is decriminalized, and the law allows minors and victims of rape to terminate a pregnancy without the requirement for 
a court order ${ }^{21}$.

The main limitation of this evaluation is that it was conducted as part of an ongoing program, and retrospective data was limited by the available information in the records. And even though the methodology was followed rigorously, the resulting data from the retrospective period could not answer some important questions. Another caveat is that, despite additional layers of permissions sought from the relevant institutions including RNP and the MOJ, due to time constraints, the scope of data collection was limited to health facilities and intermediate courts. It is possible that this evaluation might have missed some other applications for court orders, which could have been submitted to courts at other levels, to the police, prosecution offices or entities not included in the scope of this evaluation. However, it is safe to assume that there was only one approved court order identified in this evaluation. The team of researchers including $\mathrm{MOH}$ and MOJ representatives would have been likely to identify any other approvals for pregnancy termination if it had been issued in the districts searched - as it was clear that getting a court order for abortion makes a very high profile case in Rwanda.

\section{Conclusion}

The operationalization of the PC-2012 has made significant contributions to make abortion safer in Rwanda through legal reform, development of national protocol and training materials, capacity building through training of health providers, community leader involvement, and supporting a forum for open discussion and building evidence. As such, Rwanda's efforts should be inspiring for other countries in the region with similar restrictive laws, with few legal exemptions. However, this evaluation demonstrated that further efforts are required to reach the goal of providing "safe abortion services to $100 \%$ of women in need and eligible according to the legal framework" per the strategic plan. Within the two and a half years of the publication of the PC-2012, not much has changed for eligible women who practically did not have access to safe abortion.

Addressing abortion stigma at the community, organizational and structural levels; working across ministry lines to integrate legal abortion and GBV services; further strengthening of service provision; and continuing collaboration and problem solving with legal and law enforcement authorities to streamline legal requirements to protect particularly young women from sexual violence and to make safe abortion a realistic option for GBV victims are some of the important next steps for a full implementation and translation of the legal framework into impact.

\section{Contribution of Authors}

Dr. Ngabo and Dr. Prata conceived the study. Dr. Hodoglugil, working with the team, was primarily responsible for the design and implementation of the study, and prepared the manuscript. Dr. Nyirazinyoye lead the qualitative data collection component. Dr. Ngoga provided guidance on the finalization of the data collection tools, implementation of the program and interpretation of results. Ms. Dushimeyezu and Mr. Kanyamanza lead data collection and supervision of the sites, and Ms. Ortega lead data analysis.

\section{Acknowledgments}

The authors would like to acknowledge Shannon Bledsoe, Allison Boiles, Pamela Norick, Calandra Park, and Dat Tran of Venture Strategies Innovations and Felix Sayingoza of Rwanda Ministry of Health for contributing to the program in Rwanda and reviewing previous versions of this assessment in the form of program reports. We thank Spencer Bugingo for his support to collect data from courts. Julie Wieland provided valuable monitoring and evaluation support during the training of data managers, as well as qualitative and quantitative data collection. We also thank the dedicated health care providers, key informants and women who participated in interviews and focus group discussions for giving us their valuable time and sharing their thoughts. The program that this manuscript is based on was partially funded by the David and Lucile Packard 
Foundation. Final thanks go to anonymous reviewers who helped improve the quality of this manuscript.

\section{References}

1. National Institute of Statistics of Rwanda. Available at: http://www.statistics.gov.rw/. Accessed date: 24 December 2016.

2. MDG Monitor. Tracking the Millennium Development Goals. MDG Progress Report of Africa in 2015. Available at: http://www.mdgmonitor.org/mdg-progress-reportafrica/. Access date: 24 December 2016.

3. National Institute of Statistics of Rwanda, Ministry of Health of Rwanda, ICF International. Rwanda Demographic and Health Survey 2014-15: Final Report. Available at: https://dhsprogram.com/publications/publicationfr316-dhs-final-reports.cfm. Access date: 26 December 2016.

4. Basinga P, Moore A, Singh S, Carlin E, Birungi F, Ngabo F. Abortion Incidence, Morbidity and Postabortion Care in Rwanda. Studies in Family Planning; 2012. 43(1):11-20.

5. Vlassof M, Musange SF, Kalisa IR, Ngabo F, Sayingoza F, Singh S, Bankole A. The Health System Cost of Postabortion Care in Rwanda. Health Policy and Planning; 2015. 30 (2):223-233.Available at: http://heapol.oxfordjournals.org/content/30/2/223. Access date: 26 December 2016.

6. Sedgh, G, Bearak J, Singh S, Bankole A, Popinchalk A, Ganatra B, Rossier C, Gerdts C, Tunçalp Ö, Johnson BR, Johnston HB, Alkema L. Abortion incidence between 1990 and 2014: global, regional, and subregional levels and trends. The Lancet; 2016. 388 (10041):258-267.

7. Guttmacher Institute. Abortion in Africa. Incidence and Trends. Fact Sheet. 2016. Available at: https://www.guttmacher.org/fact-sheet/factsabortion-africa. Access date: 24 December 2016.

8. Okonofua, F.E. Contribution of anti-abortion laws to maternal mortality in developing countries. Expert Review in Obstetrics and Gynecology. 2008, 3(2), 147-149. Available at: http://www.tandfonline.com/doi/full/10.1586/17474 108.3.2.147. Access date: 24 December 2016.

9. Singh, S., Sedgh, G., Bankole, A., Hussain, R., and London, S. Making Abortion Services Accessible in the Wake of Legal Reforms: A Framework and Six Case Studies; 2012 (p. 50). New York: The Guttmacher Institute. Available at: https://www.guttmacher.org/sites/default/files/repor t_pdf/abortion-services-laws_0.pdf. Accessed date: 24 December 2016.

10. Umuhoza C, Oosters B, van Reeuwijk M, Vanwesenbeeck I. Advocating for safe abortion in
Rwanda: how young people and the personal stories of young women in prison brought about change. Reproductive Health Matters; 2013. 21(41):49-56. Available at: http://dx.doi.org/10.1016/S09688080(13)41690-7.

11. Republic of Rwanda. Organic Law instituting the Penal Code $\mathrm{N}^{\circ}$ 01/2012/OL of 02/05/2012, June 14, 2012. Available at: http://www.police.gov.rw/uploads/tx download/Official_Gazette_no_Special_of_14.06.2 012-4.pdf. Access date: 26 December 2016.

12. African Union. Protocol to the African Charter on Human and Peoples' Rights on the Rights of Women in Africa (Maputo Protocol), 11 July 2003. Available at: http://www.achpr.org/instruments/ women-protocol/8. Access date:26 December 2016.

13. Rwanda Ministry of Health. The Second Rwanda Road Map to Accelerate the Reduction of Maternal and Neonatal Morbidity and Mortality (2013-2018). 2012.

14. Rwanda Ministry of Health, Venture Strategies Innovations (VSI). Expanding Access to Postabortion Care Services in Rwanda, 2013. Available at:.http://bixby.berkeley.edu/publication/expandingaccess-to-postabortion-care-services-in-rwanda/. Access date: 26 December 2016.

15. Rwanda Ministry of Health, Venture Strategies Innovations (VSI). Expanding Comprehensive Postabortion Care Services in Gisagara. 2013.

16. Rwanda Ministry of Health. Guidance Document to Operationalize the Exemptions for Abortion in the Penal Code of 2012 (2014-2018). 2014.

17. Rwanda Ministry of Health. National Protocol for the Operationalization of Exemptions for Abortion in the Penal Code of 2012. 2014.

18. Rwanda Ministry of Gender and Family Promotion. National Scale up of Isange One Stop Center (IOSC) Joint Program. 2015. Available at: http://www.migeprof.gov.rw/index.php?id=169\&L= 1. Access date: 26 December 2016.

19. Kumar A, Hessini L, Mitchell EMH

Conceptualising abortion stigma. Culture Health and Sexuality; 2009, 11(6): 625-639. Available at: http://www.tandfonline.com/doi/abs/10.1080/13691 050902842741 ?journalCode $=$ tchs20. Access date: 26 December 2016.

20. Yegon EK, Kabanya PM, Echoka E, Osur J. Understanding Abortion-related Stigma and Incidence of Unsafe Abortion: Experiences from Community Members in Machakos and Trans Nzoia Counties Kenya. The Pan African Medical Journal; 2016, 24:258. Available at: http://www.panafrican-medjournal.com/content/article/24/258/pdf/258.pdf. Access date: 26 December 2016.

21. Guttmacher Institute. Facts on Unintended Pregnancy and Abortion in Ethiopia. 2010. Available at: https://www.guttmacher.org/pubs/FBUP-Ethiopia.pdf Access date: 26 December 2016. 